\title{
Time for the Human Screenome Project
}

Byron Reeves, Thomas Robinson \& Nilam Ram

\section{To understand how people use digital media, researchers need to move beyond screen time and capture everything we do and see on our screens.}

$\mathbf{T}$ here has never been more anxiety about the effects of our love of screens - which now bombard us with social-media updates, news (real and fake), advertising and blue-spectrum light that could disrupt our sleep. Concerns are growing about impacts on mental and physical health, education, relationships, even on politics and democracy. Just last year, the World Health Organization issued new guidelines about limiting children's screen time; the US Congress investigated the influence of social media on political bias and voting; and California introduced a law (Assembly Bill 272) that allows schools to restrict pupils' use of smartphones.

All the concerns expressed and actions taken, including by scientists, legislators, medical and public-health professionals and advocacy groups, are based on the assumption that digital media - in particular, social media - have powerful and invariably negative effects on human behaviour. Yet so far, it has been a challenge for researchers to demonstrate empirically what seems obvious experientially. Conversely, it has also been hard for them to demonstrate that such concerns are misplaced.

A major limitation of the thousands of studies, carried out over the past decade or so, of the effects of digital media is that they do not analyse the types of data that could reveal exactly what people are seeing and doing on their screens - especially in relation to the problems that doctors, legislators and parents worry most about. Most use self-reports of 'screen time'. These are people's own estimates of the time they spend engaging with screens or with platforms that are categorized as 'smartphone', 'television', 'social media', 'political news' or 'entertainment media'. Yet today's media experiences defy such simplistic characterization: the range of content has become too broad, patterns of consumption too fragmented ${ }^{1}$, information diets too idiosyncratic ${ }^{2}$, experiences too interactive and devices too mobile.

Policies and advice must be informed by accurate assessments of media use. These should involve moment-by-moment capture of what people are doing and when, and machine analysis of the content on their screens and the order in which it appears.

Technology now allows researchers to record digital life in exquisite detail. And thanks to shifting norms around data sharing, and the accumulation of experience and tools in fields such as genomics, it is becoming easier to collect data while meeting expectations and legal requirements around data security and personal privacy.

We call for a Human Screenome Project - a collective effort to produce and analyse recordings of everything people see and do on their screens.

\section{Screen time}

According to a 2019 systematic review and meta-analysis ${ }^{3}$, over the past 12 years, 226 studies have examined how media use is related to psychological well-being. These studies consider mental-health problems such as anxiety, depression and thoughts of suicide, as well as degrees of loneliness, life satisfaction and social integration.

The meta-analysis found almost no systematic relationship between people's levels of exposure to digital media and their well-being. But almost all of these 226 studies used responses to interviews or questionnaires about how long people had spent on social media, say, the previous day.

The expectation is that if someone reports being on Facebook a lot, then somewhere among all those hours of screen time are the ingredients that influence well-being, for

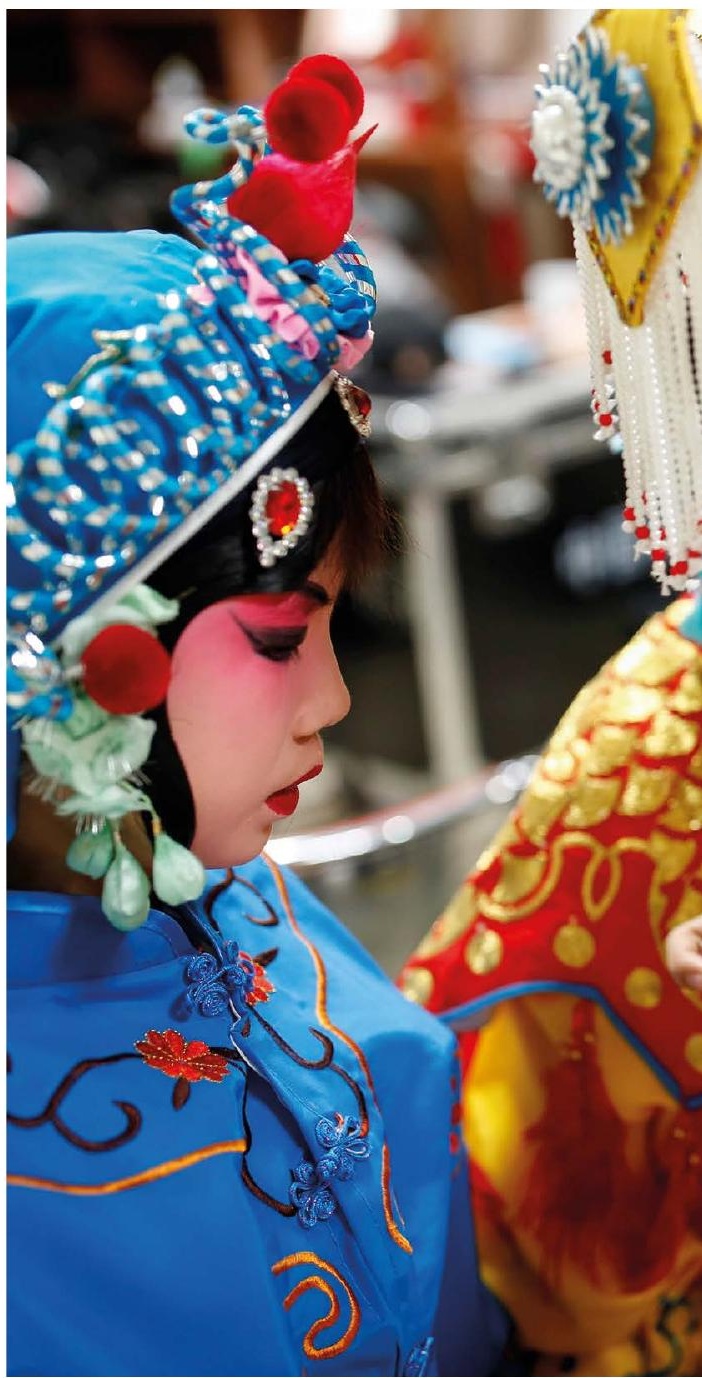

better or worse. But 'time spent on Facebook' could involve finding out what your friends are doing, attending a business meeting, shopping, fundraising, reading a news article, bullying, even stalking someone. These are vastly different activities that are likely to have very different effects on a person's health and behaviour.

Another problem is that people are unlikely to recollect exactly when they did what $^{4,5}$. Recent studies that compared survey responses with computer logs of behaviour indicate that people both under- and over-report media exposure - often by as much as several hours per day ${ }^{6-8}$. In today's complex media environment, survey questions about the past month or even the past day might be almost useless. How many times did you look at your phone yesterday?

The US National Institutes of Health (NIH) is 


\section{UNDERTHEMICROSCOPE}

Recordings of smartphone use by two 14-year-olds living in the same northern California community reveal what can be learnt from a fine-grained analysis of media use (see 'All in the details').

Dose. A typical question that researchers might ask is whether study participants are 'heavy' or 'light' phone users. Both adolescents might have characterized their phone use as 'substantial' had they been asked the usual survey questions. Both might have reported that they used their smartphones 'every day' for ' 2 or more hours' each day, and that looking at their phones was the first thing they did each morning and the last thing they did every night.

But detailed recordings of their actual phone use over 3 weeks in 2018 highlight dramatic differences ${ }^{2}$. For participant $A$, median use over the 3 weeks was 3.67 hours per day. For participant B, it was 4.68 hours, an hour (27.5\%) more.

Pattern. The distribution of time spent using phones during the day differed even more. On average, participant A's time was spread over 186 sessions each day (with a session defined as the interval between the screen lighting up and going dark again). For $A$, sessions lasted 1.19 minutes on average. By contrast, participant B's time was spread over 26 daily sessions that lasted, on average, 2.54 minutes. So one of the adolescents turned their phone on and off seven times more than the other, using it in bursts that were about one-third the length of the other's sessions.

These patterns could signal important psychological differences. Participant A's days were more fragmented, maybe

instance, that levels of well-being are related to how fragmented people's use of media is, or the content that they engage with. Differences in brain structure might be related to how quickly people move through cycles of production and consumption of content. Differences in performance in cognitive tasks might be related to how much of a person's multitasking involves switching between content (say, from politics to health) and applications (social media to games), and how long they spend on each task before switching.

\section{The Human Screenome Project}

So, how can we do better? What's needed is a collective effort to record and analyse everything people see and do on their screens, the order in which that seeing and doing occurs, and the associated metadata that are indicating issues with attentional control, or perhaps reflecting an ability to process information faster.

Interactivity. Both adolescents spent time creating content as well as consuming it. They wrote text messages, recorded photos and videos, entered search terms and so on. On a questionnaire, both might have reported that they posted original material 'sometimes' or maybe 'often'. But the screenshot data reflect patterns of interactivity that would be almost impossible for them to recall accurately.

Participant A spent $2.6 \%$ of their screen time in production mode, creating content evenly throughout the day and usually within social-media apps. By contrast, participant B spent $7 \%$ of their total screen time producing content (and produced 2.5 times more). But they did so mainly in the evening while watching videos.

Content. During the 3 weeks, participant A engaged with 26 distinct applications. More than half of these (53.2\%) were social-media apps (mostly Snapchat and Instagram). Participant $B$ engaged with 30 distinct applications, mostly YouTube $(50.9 \%$ of the total).

Zooming deeper into specific screen content reveals even more. For participant B, on average, $37 \%$ of the screenshots for a single day included food - pictures of food from various websites, photos of B's own food, videos of other people eating or cooking, and food shown in a game involving the running of a virtual restaurant.

In a survey, both adolescents might have reported that they used 'a lot' of apps, and might have given the names of some of them. But the content of their media diets would be impossible to capture. B.R. et al.

available from the software and sensors built into digital devices (for instance, on time of day, location, even keystroke velocity).

In any one screenome, screenshots are the fundamental unit of media use. But the particular pieces or features of the screenome that will be most valuable will depend on the question posed - as is true for other 'omes'. If the concern is possible addiction to mobile devices, then arousal responses (detected by a change in heart rate, say) associated with the first screen experienced during a session might be important to measure. If the concern is the extent to which social relationships dictate how political news is evaluated, then the screenshots that exist between 'social' and 'political' fragments in the screenome sequence might be the crucial data to analyse. (News items flagged by a close friend might be perceived as more trustworthy than the same news obtained independently, for example.)

How can researchers get access to such high-resolution data? And how can they extract meaning from data sets comprising millions of screenshots?

One option is for investigators to collaborate with the companies that own the data, and that have already developed sophisticated ways to monitor people's digital lives, at least in certain domains, such as Google, Facebook, Amazon, Apple and Microsoft. The Social Science One programme, established in 2018 at Harvard University in Cambridge, Massachusetts, involves academics partnering with companies for exactly this purpose $^{12}$. Researchers can request to use certain anonymized Facebook data to study social media and democracy, for example.

Largely because of fears about data leaks or study findings that might adversely affect business, such collaborations can require compromises in how research questions are defined and which data are made available, and involve lengthy and legally cumbersome administration. And ultimately, there is nothing to compel companies to share data relevant to academic research.

To explore more freely, academics need to collect the data themselves. The same is true if they are to tackle questions that need answers within days - say, to better understand the effects of a terrorist attack, political scandal or financial catastrophe.

Thankfully, Screenomics and similar platforms are making this possible.

In our experience, people are willing to share their data with academics. The harder problem is that collecting screenomics data rightly raises concerns about privacy and surveillance. Through measures such as encryption, secure storage and de-identification, it is possible to collect screenomes with due attention to personal privacy. (All our project proposals are vetted by university institutional review boards, charged with protecting human participants.) Certainly, social scientists can learn a lot from best practice in the protection and sharing of electronic medical records ${ }^{13}$ and genomic data.

Screenomics data should be sifted using a gamut of approaches - from deep-dive qualitative analyses to algorithms that mine and classify patterns and structures. Given how quickly people's screens change, studies should focus on the variation in an individual's use of media over time as much as on differences between individuals and groups. Ultimately, researchers will be able to investigate moment-by-moment influences on physiological and psychological states, the sociological dynamics of interpersonal and group relations over days and weeks, and even cultural and historical changes that accrue over months and years. 


\section{ALL IN THE DETAILS}

Recordings of screenshots every five seconds reveal substantial differences in how

two adolescents use their smartphones over 21 days (see 'Under the microscope').

Comics Video players and editors Communications

Photography Social Games Education Study $\square$ Tools $\square$ Music and audio $\Delta$ Creating content (not shown on the larger figure)

\section{Participant A}

Participant A's time was spread over 186 sessions per day (with a session defined as the interval between the screen lighting up and going dark again). Each session lasted 1.19 minutes on average.

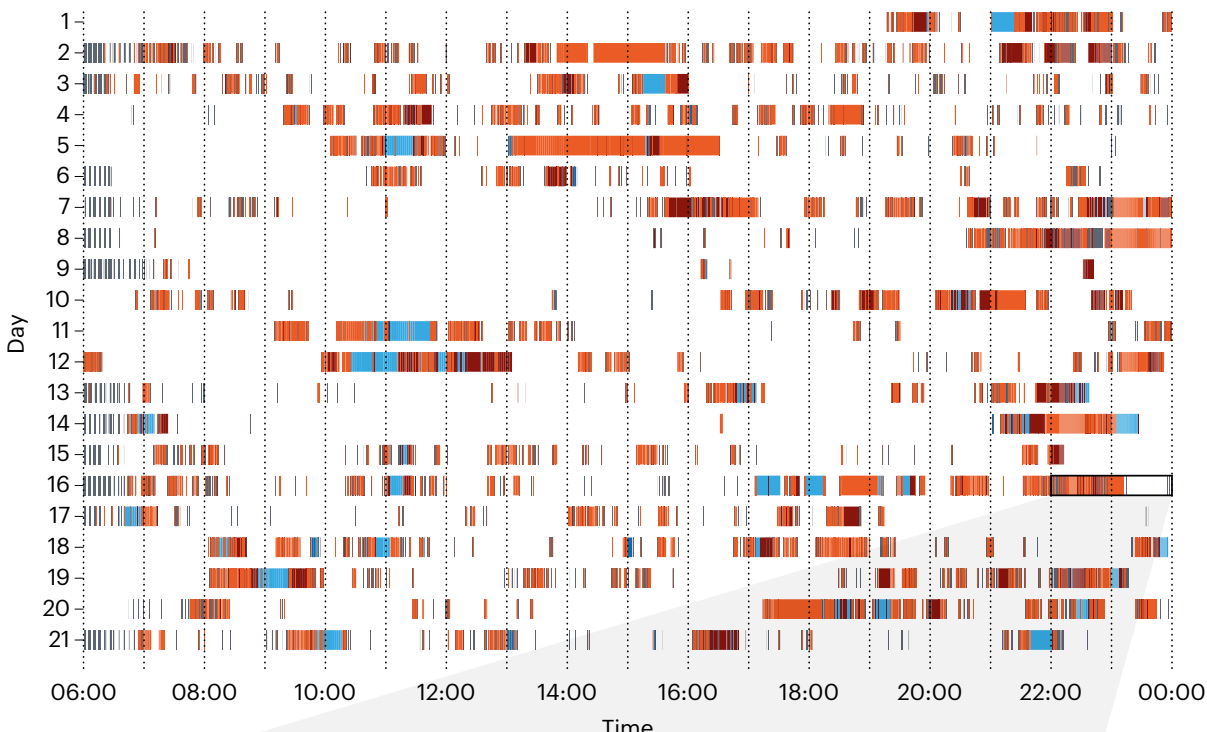

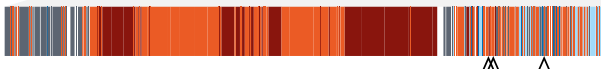

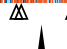

Zooming in on 2 hours of participant A's activity on day 16 reveals more about how they spent their time. More than half of the apps that A engaged with were

types of social media (mostly Snapchat and Instagram).

Participant B

Participant B's time was spread over 26 sessions per day, lasting 2.54 minutes on average.

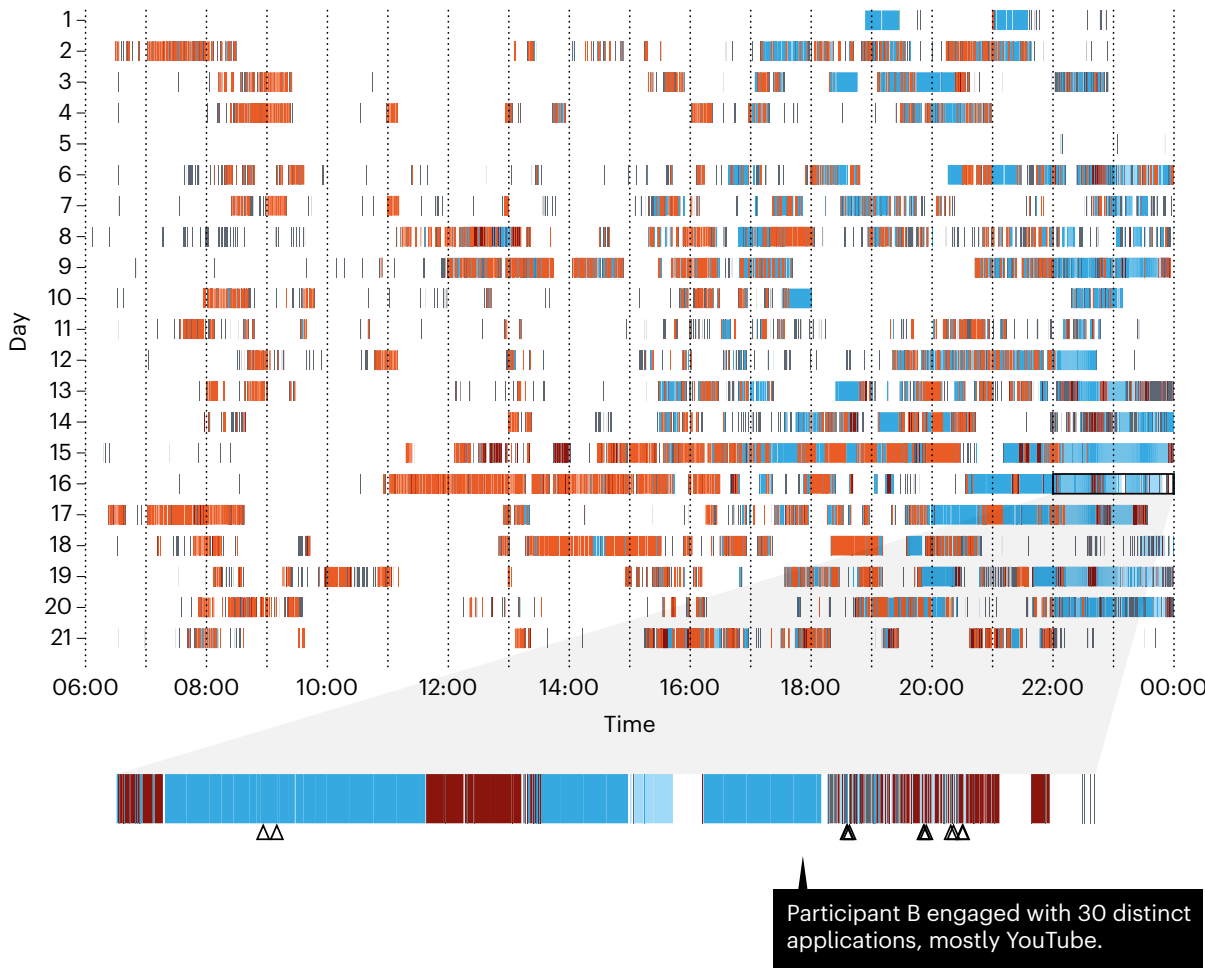

Some might argue that screenomics data are so fine-grained that they invite researchers to focus on the minutiae rather than the big picture. We would counter that today's digital technology is all about diffused shards of experience. Also, through the approach we propose, it is possible to zoom in and out, to investigate how the smallest pieces of the screenome relate to the whole. Others might argue that even with this better microscope, we will not find anything significant. But if relationships between the use of media and people's thoughts, feelings and behaviours continue to be weak or non-existent, at least we could have greater confidence as to whether current concerns are overblown.

The approach we propose is complex, but no more so than the assessment of genetic predictors of mental and physical states and behaviours. Many years and billions of US dollars have been invested in other 'omics' projects. In genomics, as in neuroscience, planetary science and particle physics, governments and private funders have stepped up to help researchers gather the right data, and to ensure that those data are accessible to investigators globally. Now that so much of our lives play out on our screens, that strategy could provejust as valuable for the study of media.

\section{The authors}

Byron Reeves is a professor of communication at Stanford University, California, USA.

Thomas Robinson is a professor of child health, paediatrics and medicine, and director of the Stanford Solutions Science Lab at Stanford University, California, USA. Nilam Ram is a professor of human development and psychology, and director of the Quantitative Developmental Systems Group at Pennsylvania State University, University Park, Pennsylvania, USA.

e-mails: reeves@stanford.edu; tom.robinson@ stanford.edu; nur5@psu.edu

1. Yeykelis, L., Cummings, J. J. \& Reeves, B. J. Commun. 64 167-192 (2014).

2. Ram, N. et al. J. Adolesc. Res. 35, 16-50 (2020).

3. Hancock, J., Liu, X., French, M., Luo, M. \& Mieczkowski, $\mathrm{H}$. Social media use and psychological well-being: a meta-analysis. Paper presented at 69th Annu. Conf. Int. Commun. Assoc., Washington DC (2019).

4. Prior, M. Polit. Commun. 30, 620-634 (2013).

5. Niederdeppe, J. Commun. Meth. Measures 10, 170-172 (2016).

6. Araujo, T., Wonneberger, A., Neijens, P. \& de Vreese, C. Commun. Meth. Measures 11, 173-190 (2017).

7. Naab, T. K., Karnowski, V. \& Schlütz, D. Commun. Meth Measures 13, 126-147 (2019).

8. Junco, R. Comp. Human Behav. 29, 626-631 (2013).

9. Paulus, M. P. et al. Neuroimage 185, 140-153 (2019).

10. Yeykelis, L., Cummings, J. J. \& Reeves, B. Media Psychol. 21, 377-402 (2018).

11. Reeves, B. et al. Human-Comp. Interact. 34, 1-52 (2019).

12. King, G., \& Persily, N. PS: Polit. Sci. Polit. https://doi. org/10.1017/S1049096519001021 (2019).

13. Parasidis, E., Pike, E. \& McGraw, D. N. Engl. J. Med. 380 , 1493-1495 (2019). 